\title{
Effect of Textured Foot Orthoses on the Ground Reaction Force Components in the Older Adults During Walking
}

\author{
Aydin Valizadeorang ${ }^{1, *}$ (i), Arefeh Mokhtari-Malekabadi ${ }^{2}$ (D), AmirAli Jafarnezhadgero \\ ${ }^{1}$ Assistant Prof, Department of Physical Education and Sport Sciences, Faculty of Educational Sciences and Psychology, University \\ of Mohaghegh Ardabili, Ardabil, Iran \\ ${ }^{2}$ MSc in Sport Biomechanics, Faculty of Educational Sciences and Psychology, University of Mohaghegh Ardabili, Ardabil, Iran \\ * Corresponding author: Aydin Valizadeorang, Assistant Prof, Department of Physical Education and Sport Sciences, Faculty of \\ Educational Sciences and Psychology, University of Mohaghegh Ardabili, Ardabil, Iran. E-mail: jteymour@gmail.com \\ How to Cite this Article: \\ Valizadeorang A, Mokhtari-Malekabadi A, Jafarnezhadgero AA. Effect of Textured Foot Orthoses on the Ground Reaction Force \\ Components in the Older Adults During Walking. Iran J Rehab Res Nurs. 2020;7(1):23-32. \\ DOI: 10.29252/ijrn.7.1.23
}

Received: 30 May 2019 Accepted: 09 Nov 2019

Keywords:

Walking

Kinetic

Orthoses of Foot

Older Adult

(C) 2020 Iranian Journal of

Rehabilitation Research in Nursing

\begin{abstract}
Introduction: With aging, the pattern of walking is altered, and the person's ability to walk will be decreased. Also, with the beginning of the aging process, the coordination of the postural control system was disturbed and lead to an unstable condition. A small biomechanical change in the lower limb is significant on postural control. The use of textured foot orthoses leads to improved efficiency and increased comfort. The purpose of this study was to investigate the effect of textured foot orthoses on ground reaction force components in older adults during walking.

Methods: The present study was of a semi-experimental and laboratory type. Twentyone older adults with available sampling were volunteered to participate in this study. Ground reaction force data were recorded by the Beretc force plates (Beretc CorporationColumbus, $\mathrm{OH}$ ) during walking without foot orthoses, walking with small and large textured foot orthoses. ANOVA test with repeated measures was used for statistical analysis. All analyzes were performed at the significance level of 0.05 . Results: The results showed a lower peak posterior force amplitude during the heel contact phase while walking with small textured foot orthoses than that walking without foot orthoses $(P=0.003)$. Time to the peak of vertical force during the heel contact phase was decreased while walking with large textured foot orthoses compared with walking without foot orthoses condition $(P=0.048)$. Also, time to peak of lateral force during the heel contact phase was increased while walking with small textured foot orthoses compared with walking without foot orthoses condition $(P=0.021)$.

Conclusions: Using small textured foot orthoses compared to the large textured ones improved most of the ground reaction of elders. However, both small and sizeable textured foot orthoses were not useful on torsion stresses on the body.
\end{abstract}

\section{Extended Abstract}

\section{OBJECTIVE}

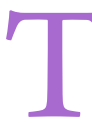

he progression of ground reaction forces through the lower limbs during walking occurs in a distal to the proximal manner. When walking, the plantar part of the foot is the first point of contact between the body and the external environment, providing sensory data to the central nervous system for postural control and locomotion.
With aging, the pattern of walking is altered, and the person's ability to walk will be decreased. Also, with the beginning of the aging process, the coordination of the postural control system was disturbed and lead to an unstable condition. A small biomechanical change in the lower limb is significant in postural control. The use of textured 
foot orthoses leads to improved efficiency and increased comfort. The treatment of foot with textured foot orthoses might also help to reduce the ground reaction forces, vertical loading rate, impulses, and free moment which may, in turn, reduce the possibility of anterior cruciate ligament injury, patellofemoral pain syndrome, and other disorders. The purpose of this study was to investigate the effect of textured foot orthoses on ground reaction force components in older adults during walking.

\section{MATERIAL AND METHODS}

The present study was of a semi-experimental and laboratory type. Twenty-one older adults with available sampling were volunteered to participate in this study. Ground reaction force data were recorded by the Beretc force plates (Beretc Corporation Columbus, $\mathrm{OH}$ ) during walking without foot orthoses, walking with small and large textured foot orthoses. Kinetic data were filtered using a fourth-order low-pass Butterworth filter with a cutoff frequency of $20 \mathrm{~Hz}$. All data were normalized to the stance phase with heel contact to toe-off corresponding to $100 \%$. Parameters that were used for further statistical analyses included peak values in three-dimensional ground reaction forces and time to peak of the mediolateral ( $\mathrm{Fx})$, anteroposterior (Fy), and vertical $(\mathrm{Fz})$ ground reaction force axes. Forces were normalized to body weight (BW), and the corresponding timing was expressed as mili second. Vertical loading rates were computed as the average slope from $0 \%$ to $100 \%$ of the first peak of vertical ground reaction force. Data were tested for normal distribution using the Shapiro-Wilk test. Homogeneity of variance was assessed using the Levene's test and variance ratios. Data were presented in the form of means and standard deviations if the normal distribution of data was given. ANOVA test with repeated measures was used for statistical analysis. All analyzes were performed at the significance level of 0.05 .

\section{RESULTS}

The results showed lower peak posterior force amplitude during the heel contact phase while walking with small textured foot orthoses than that walking without foot orthoses $(\mathrm{P}=0.003)$. Time to the peak of vertical force during the heel contact phase was decreased while walking with large textured foot orthoses compared with walking without foot orthoses condition $(P=0.048)$. Also, time to peak of lateral force during the heel contact phase was increased while walking with small textured foot orthoses compared with walking without foot orthoses condition $(\mathrm{P}=0.021)$.

\section{CONCLUSION}

The use of small textured foot orthoses compared to the large textured ones improved most of the ground reaction force components. However, both small and sizeable textured foot orthoses were not useful on torsion stresses on the body. Textured foot orthoses may influence the quality of sensory feedback from the plantar section of the feet. They may act as a sensory filter between the feet and the external environment. Previous foot orthoses studies have demonstrated that footwear type and foot orthoses materials can influence postural stability in young adult males. These results are in line with our results. This study will be useful for the rehabilitation of older adults and can also improve walking ground reaction forces using textured foot orthoses.

\section{Ethical Considerations}

This research was confirmed by the Ethics Committee of the Medical Sciences University of Ardabil with code number IR.ARUMS.REC.1397.092. Subjects signed a consent form to participate in the study after being informed of the purpose and method of the research. Also, the information of the participants in this study was kept confidential.

\section{Funding or Supports}

There was also no funding or supports.

\section{Author's Contributions}

Mr. Aydin Valizadeorang: He did the initial idea of studying and checking the initial draft of the article, submitting, and paper revision. Ms. Arefeh MokhtariMalekabadi: She did writing and preparing the initial and final drafting, statistical analysis, and laboratory work. Mr. AmirAli Jafarnezhadgero: He did the initial design of studying and paper revision and laboratory work.

\section{Conflict of Interest}

The authors of the article do not declare any conflict of interest.

\section{Applicable Remark}

This study will be useful for the rehabilitation of older adults and can also improve walking ground reaction forces using textured foot orthoses.

\section{Acknowledgments}

We appreciate all the relevant Officials as well as all the subjects who participated in the study. 


\section{اثر كفى بافتدار بر مولفهاى نيروهاى عكسالعمل زمين در سالمندان طى راه رفتن}

آيدين ولى زاده اورنج اء* (D) عارفه مختارى ملك آبادى riD ، اميرعلى جعفرنزاد كرو

' استاديار فيزيولورى ورزشى، گروه تربيت بدنى و علوم ورزشى، دانشكده علوم تربيتى و روانشناسى، دانشخاه محقق اردبيلى، اردبيل، ايران

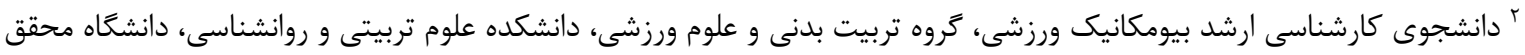
اردبيلى، اردبيل، ايران

* نويسنده مسئول: آيدين ولى زاده اورنج، استاديار فيزيولوزى ورزشى، كروه تربيت بدنى و علوم ورزشى، دانشكده علوم تربيتى و روانشناسى، دانشكاه محقق اردبيلى، اردبيل، ايران. ايميل: jteymour@gmail.com

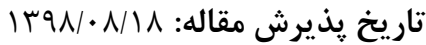

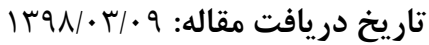

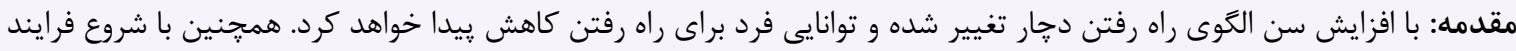

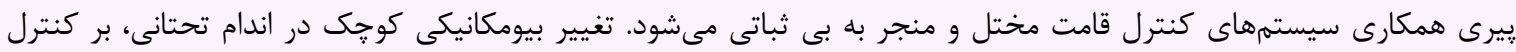

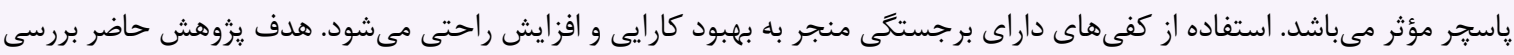

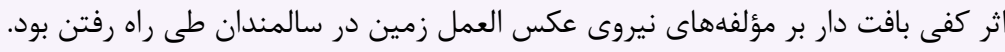

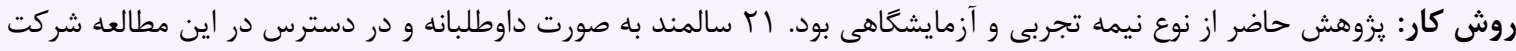

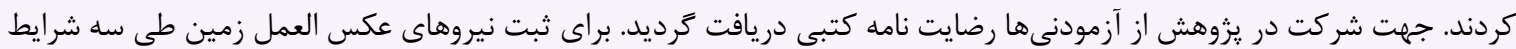

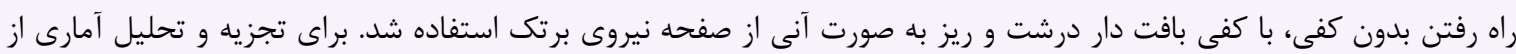

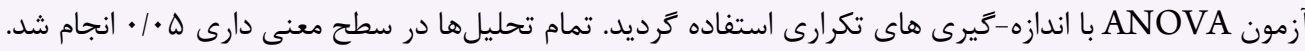

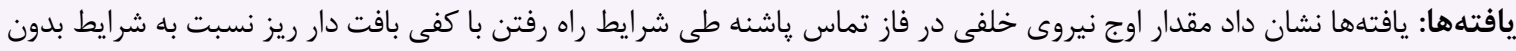

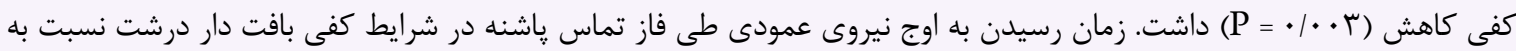

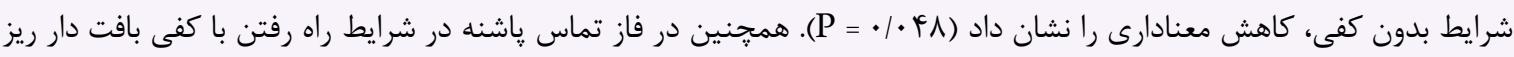

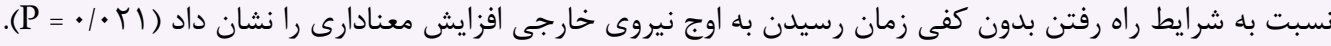

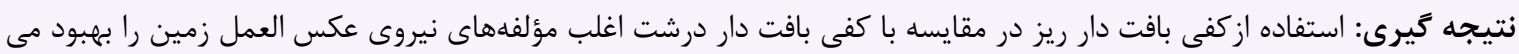

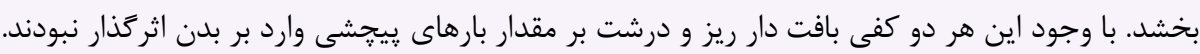
كليدوازهها: راه رفتن، كينتيك، ارتوز با، باء سالمندان

تمامى حقوق نشر براى انجمن علمى برستارى ايران محفوظ است.

مقدمه

رفتن دجار تغيير شده و توانايى فرد براى راه رفتن كم كم شروع به

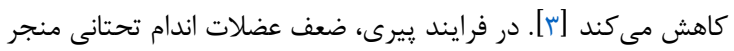

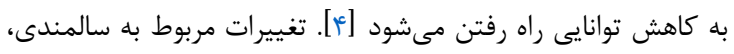

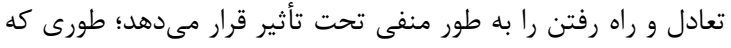

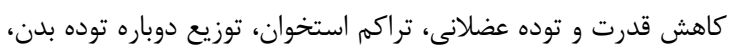
آسيب توانايى تنفسى، آتروفى مؤلفههاى سيستم عصبى مركزى تودى كه
راه رفتن يكى از مهارتهاى اساسى است كه بخش اعظم فعاليت روزمره

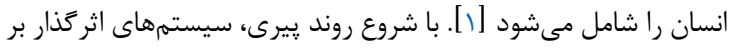
تعادل همجون سيستم سموتاسنسورى، بينايى و وستيبولار دهار

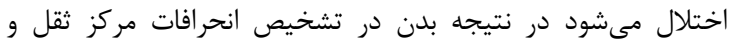

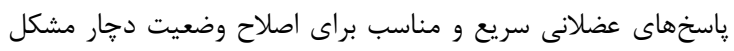
مىشود [r]. با افزايش سن و ورود فرد به دوران سالمندى، الخَّى راه 
سالمندان مىشود [.r]. مطابق يزوهش ويلسون و همكاران كه به

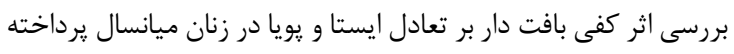

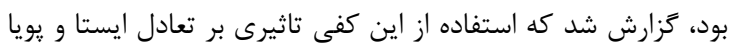

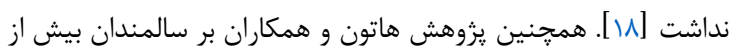

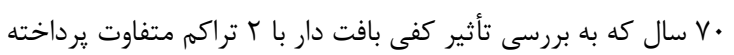

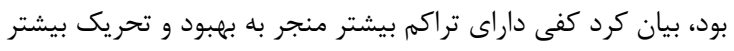

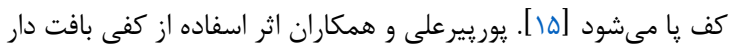

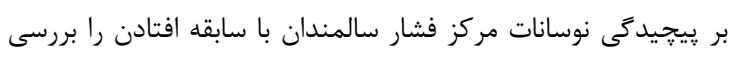

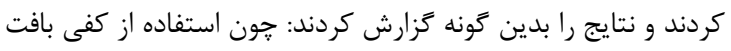

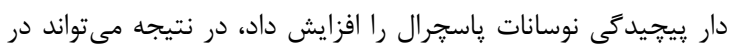

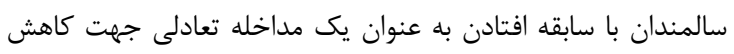

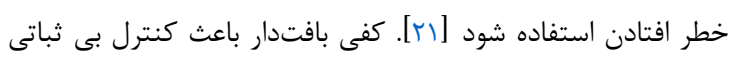

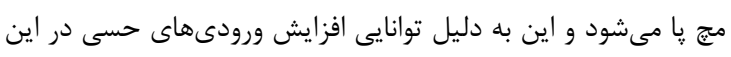

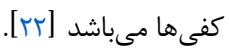
نيروهاى عكس العمل زمين، زمان رسيدن به اوج نيروهاى عكس العمل

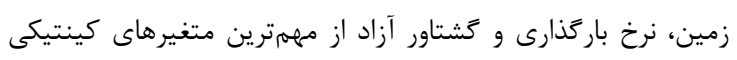

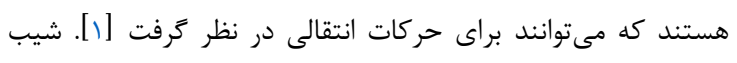

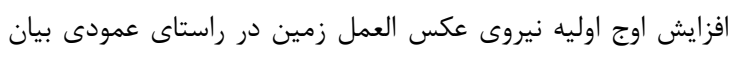

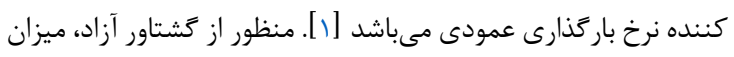

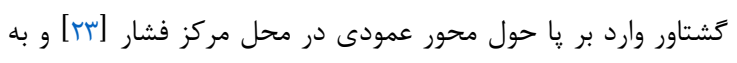

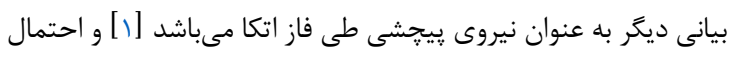

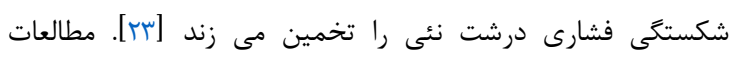

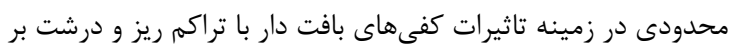

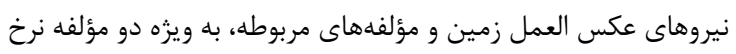

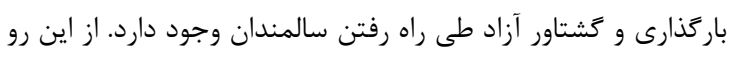

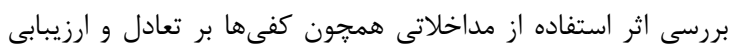

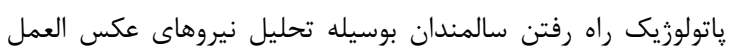

$$
\text { زمين از اهميت بالايى برخوردار است. }
$$

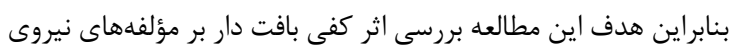

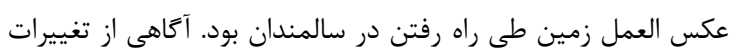

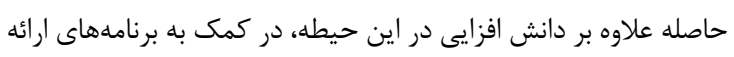
شده براى توان بخشى در سالمندان مى تواند مؤثر واقع شود.

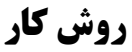

يُروهش حاضر نيمه تجربى و آزمايشًاهى بود. با استفاده از نرم افزار

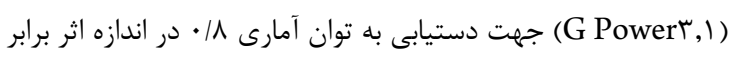

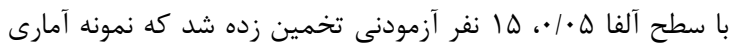

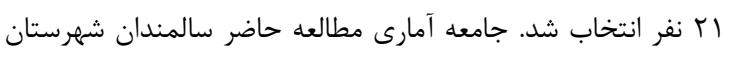

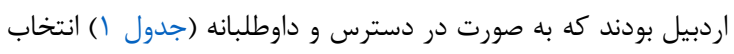

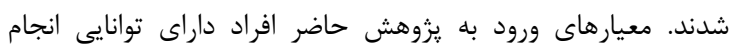

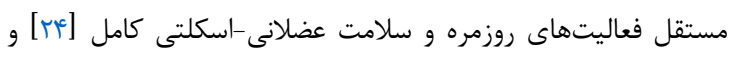

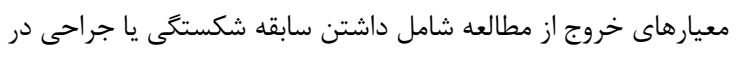

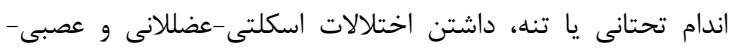

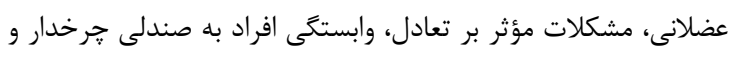

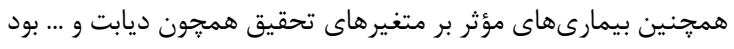

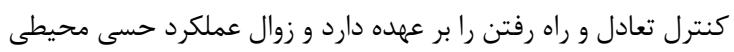

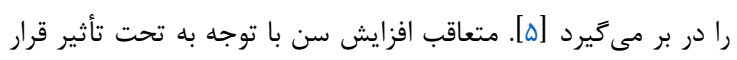

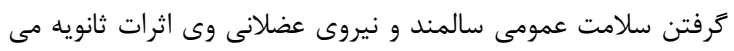

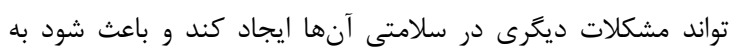

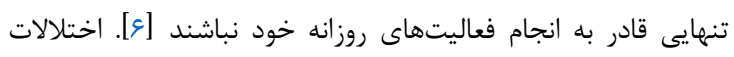

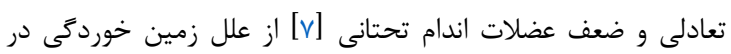

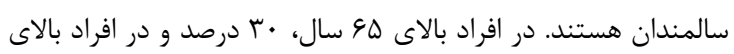

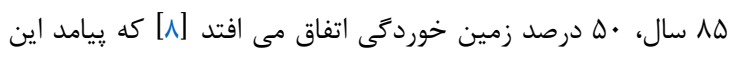

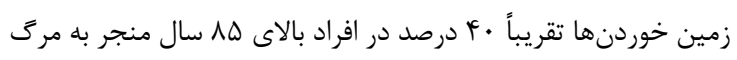

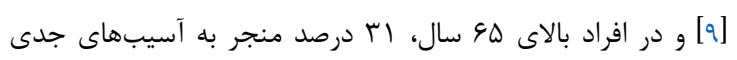

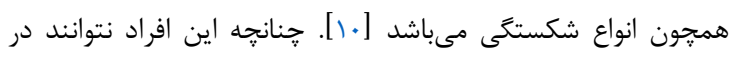

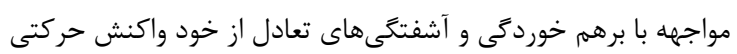

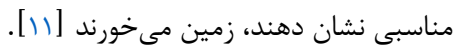

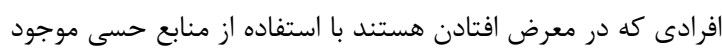

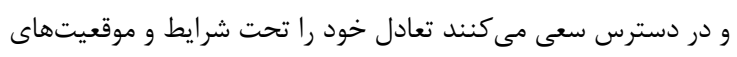

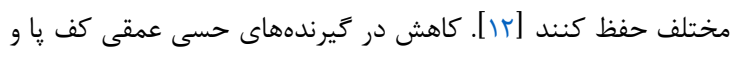

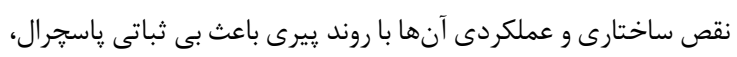

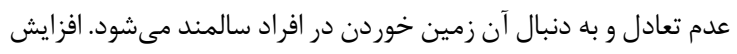

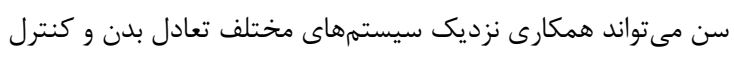

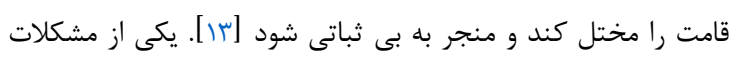

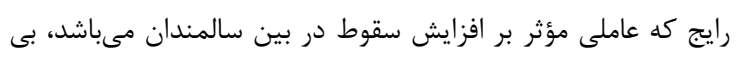

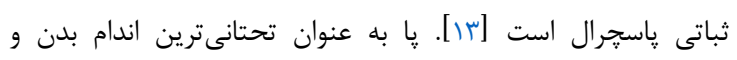

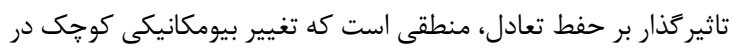

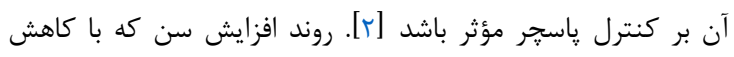

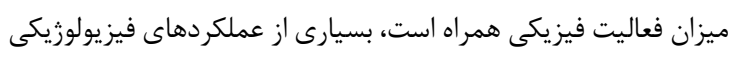

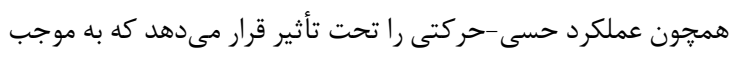

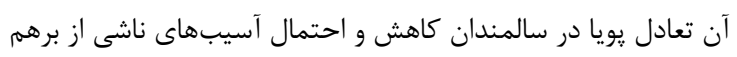

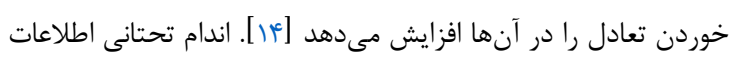

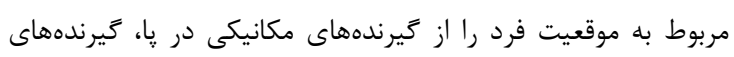

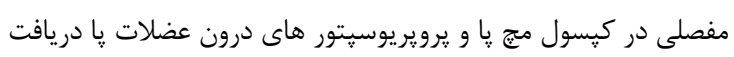

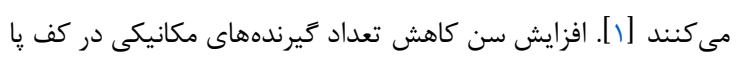

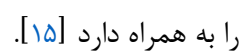

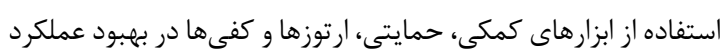

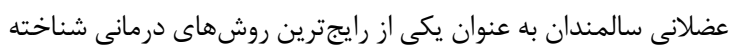

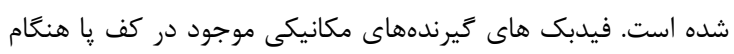

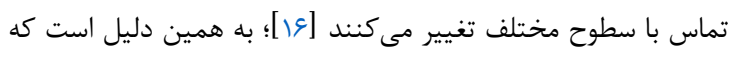

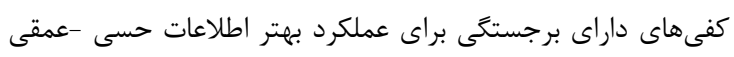

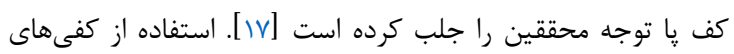

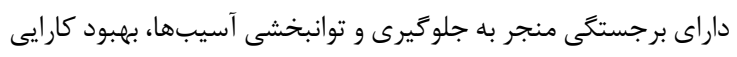

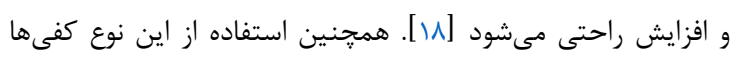

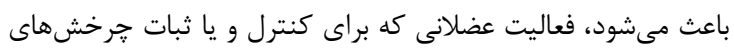

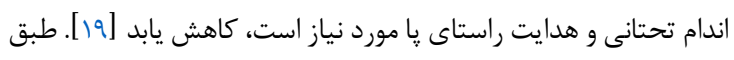

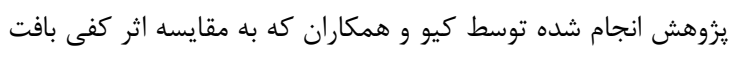

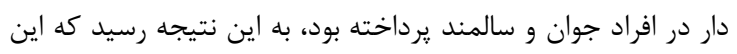

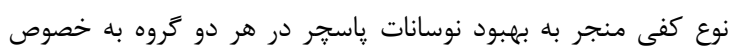




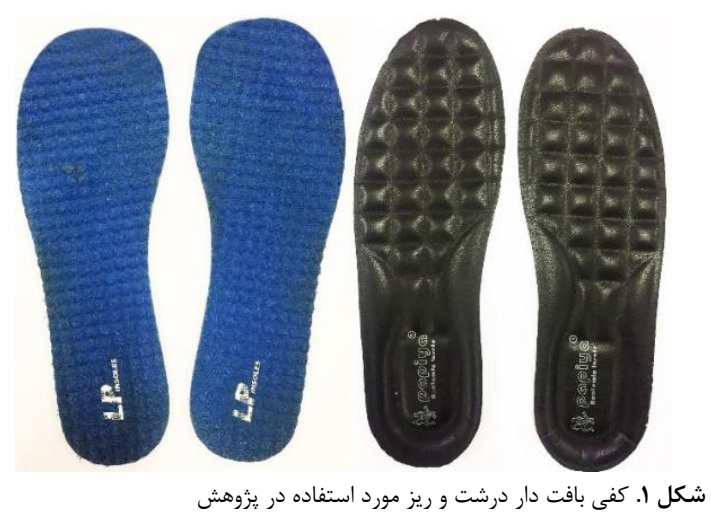

نيروهاى عكس العمل زمين (GRF) با استفاده از صفحه نيروى برتك (B) (Bertec Corporation, Columbus, OH)

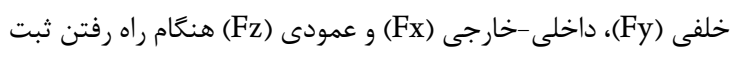

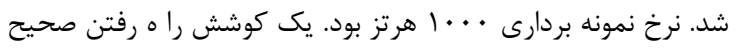

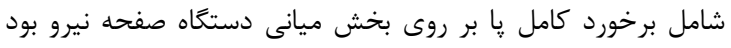

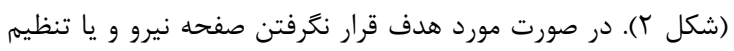

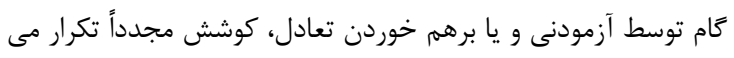

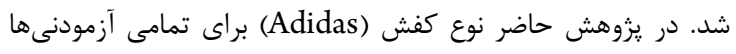

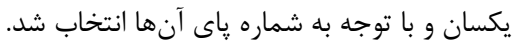

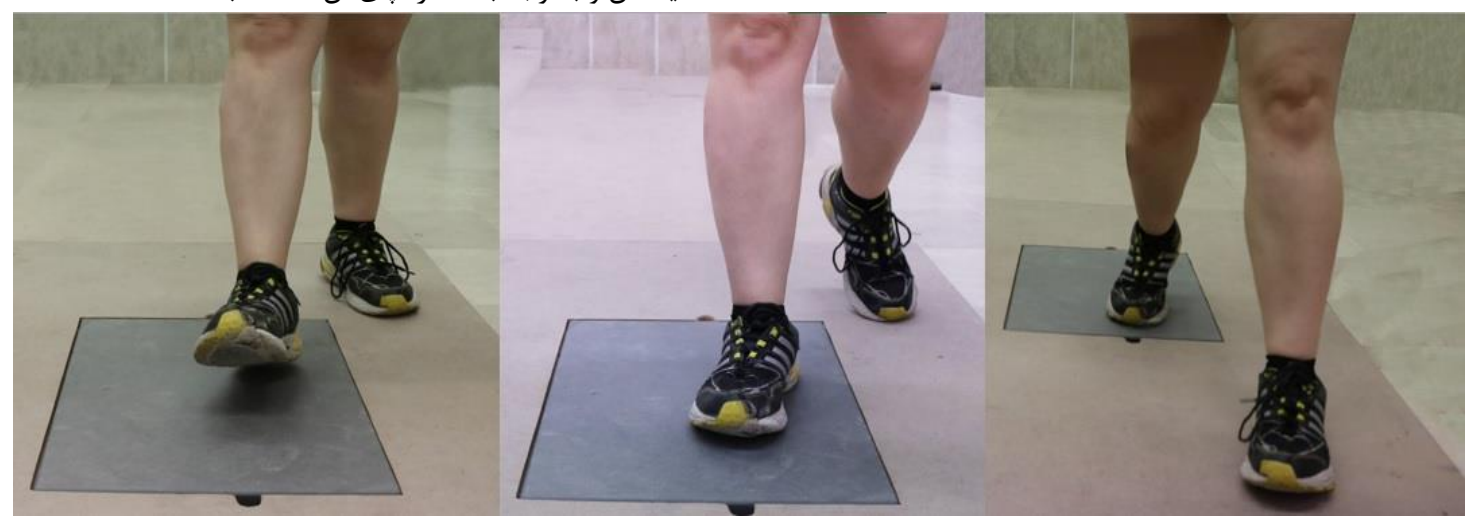

شكل r. سه فاز راه رفتن شامل برخورد ياشنه، ميانه اتكا و جدا شدن ينجه از روى دستكاه صفحه نيرو

كشتاور آزاد با استقاده از حاصلضرب وزن بدن (نيوتون) در قد (متر) نرمال شد [rv] با استفاده از آزمون شايِيرو-ويلك، نرمال بودن توزيع دادهها مورد

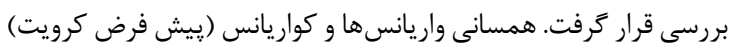

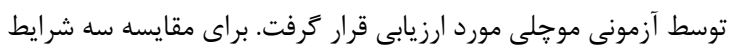

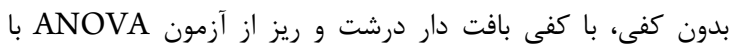

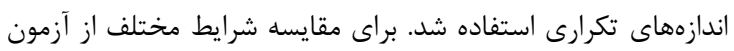

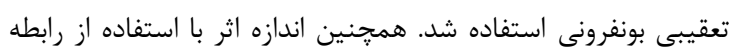
Cohen’s d

$$
D=\left(\operatorname{Mean}_{1}-\operatorname{Mean}_{2}\right) /\left(\frac{\left[\mathrm{SD}_{1}+\mathrm{SD}_{2}\right]}{2}\right)
$$

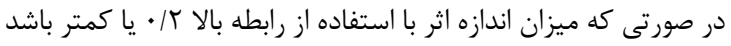

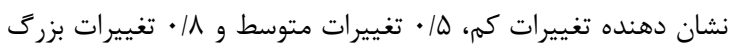

[ع]. ياى غالب آزمودنىها از طريق آزمون شوت فوتبال مشخص شد

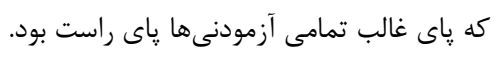

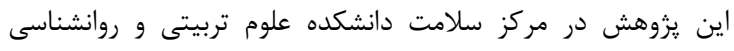

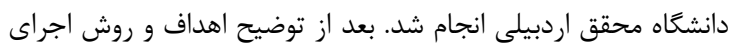

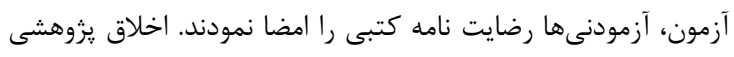

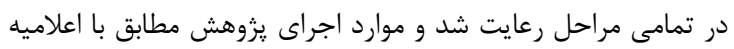

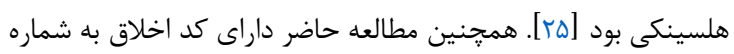

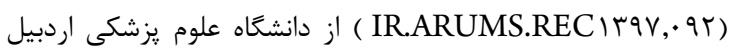

مىباشد. كفىهاى مورد استفاده در يزوهش حاضر از نوع بافت دار ريز و درشت

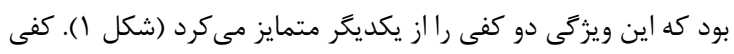

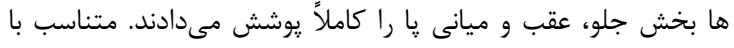

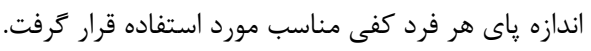

متغيرهاى نيروهاى عكس العمل زمين و زمان رسيدن به اوج نيروها

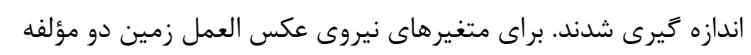

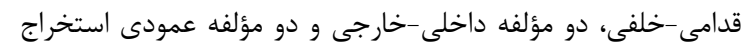

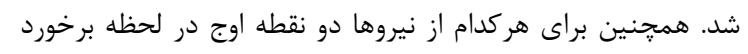

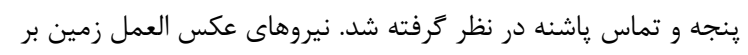

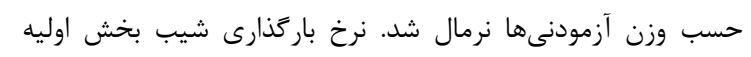

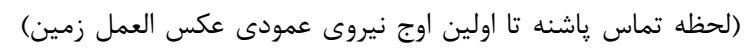
منحنى نيروى عمودى عكس العمل زمين تعريف مى شود.

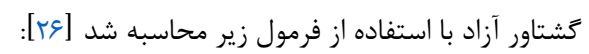

= $=\mathrm{M}_{\mathrm{z}}+\left(\mathrm{F}_{\mathrm{x}} \times \mathrm{COP}_{\mathrm{y}}\right)-\left(\mathrm{F}_{\mathrm{y}} \times \mathrm{COP}_{\mathrm{x}}\right)$

دz داخلى-خارجى COPy: مركز فشار در راستاى قدامى-خلفى مقادير 
زمان رسيدن به اوج نيروى عمودى طى فاز تماس پاشنه در شرايط

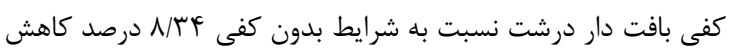

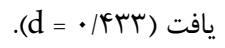

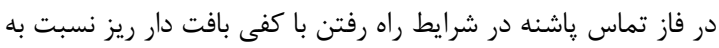

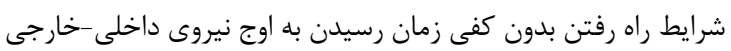

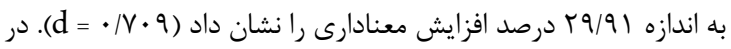

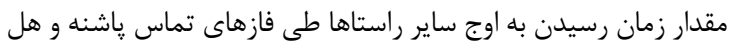

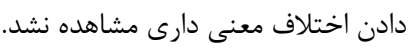

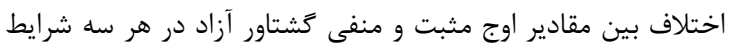

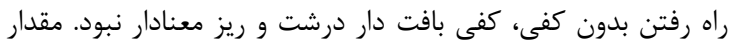

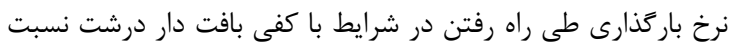

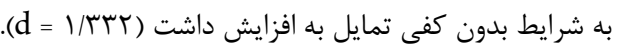

مىباشد. تمامى تحليل ها در سطح معنى دارى ه • • • و با استفاده از نرم

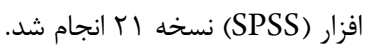

\section{كافتهها}

مشخصات شركت كنندكان در يزوهش حاضر در جدول ا نشان داده شده است. يافتهها نشان داد ميزان نيروى عمودى عكس العمل زمين طى راه رفتن

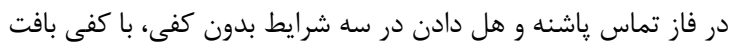

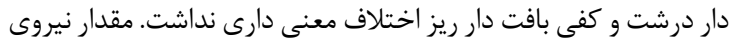

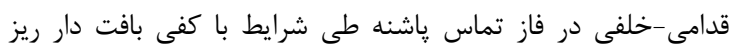

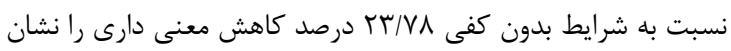

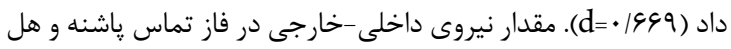
دادن در هيج كدام از شرايط ذكر شده معنى دار نبود.

\begin{tabular}{|c|c|c|c|c|}
\hline & & & ناخص توده بدن سار & جدول ا. آمار توصيفى قد \\
\hline شاخص توده بدن (كيلوكرم بر متر مربع) " & جرم (كيلوكرم)" & سن (سال)" & قد (سانتى متر)" & تعداد \\
\hline$r q / r T \Delta \pm \Delta / G K r$ & $v a / 1 \cdots \pm 1 Q / r \mu r$ & 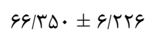 & $190 / 901 \pm 9 / 91$. & اr نفر (f/ زن و \& مرد) \\
\hline
\end{tabular}

جدول ז. اوج مؤلفهاى نيروى عكس العمل زمين (درصدى از وزن بدن) در سه بعد طى سه شرايط راه رفتن بدون كفى، با كفى بافت دار درشت و ريز

\begin{tabular}{|c|c|c|c|c|c|c|c|c|}
\hline $\begin{array}{l}\text { Partial Eta } \\
\text { Squared }\end{array}$ & $\mathbf{F}$ & 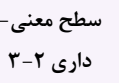 & ساح معنى - سارى ا- & سارى ا-1 معنى & كفى بافتدار ريز [r] & كفى بافتدار درشت & بدون كفى [1] "* & متغير \\
\hline & & & & & & & & نيروى عمودى \\
\hline$\cdot \|^{\circ}$ & .1 .49 & $1 / \cdots$ & $1 / \cdots$ & $1 / \cdots$ & $1 . f \pm r \Delta \cdot \pm 19 / \cdot q r$ & 1.r/Fr. $\pm 1 V / 499$ & I. r/AG. DIF/GKT & $\mathrm{FzHC}$ \\
\hline \multirow[t]{2}{*}{.1 .91} & .1994 & . Nes & $1 / \cdots$ &.$/ 91$. & I. r/Rs. $\pm I r / F \Delta$. & $1 \cdot N /|r \cdot \pm r \Delta / \Delta F|$ & 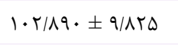 & FzPO \\
\hline & & & & & & & & نيروى قدامى -خلفى \\
\hline . $F \Delta T$ & v/Arq & $1 / \cdots$ &...$r$ &.$/ . \Delta 9$ & $-\mid F / \Delta V \cdot \pm F / K \cdot 1$ & $-|F / \pi \cdot \cdot \pm \Delta| \cdot r \Delta$ & $-11 / \gamma v \cdot \pm r / .94$ & FyHC \\
\hline \multirow[t]{2}{*}{.$/ \mu r}$. & $1 / \pi 99$ & $1 / \ldots$ & ./८99 & . $/$ kr. & $1 \Delta / V Y r \pm V / F 9 \Delta$ & $19 / 1 Y \Lambda \pm 9 / \lambda \cdot r$ & $\mid F / D Q F \pm r / \Lambda r \Delta$ & FyPO \\
\hline & & & & & & & & نيروى داخلى-خارجى \\
\hline .1 .99 & $1 / \cdot 1$ & $\cdot k \cdot 1$ & r & $\cdot r \cdot r$ & $r / \wedge 9 \Delta \pm 1 / r \Delta q$ & $r / q r \Delta \pm 1 / 911$ & $r / \Delta f \cdot \pm 1 / 9 / r$ & FxHC \\
\hline .1190 & $1 / A V V$ &.$/ \pi 11$ & $1 / \ldots$ & $\cdot \pi \Delta v$ & $-\Delta / 9 T V \pm 1 / 9 T Y$ & $-r / f \cdot f \pm r / \Delta q$. & $-g / / f V \pm \mid / A r$. & FxPO \\
\hline
\end{tabular}

جدول ب. زمان رسيدن به اوج مؤلفههاى نيروى عكس العمل زمين (ميلى ثانيه) در سه بعد طى سه شرايط راه رفتن بدون كفى، با كفى بافت دار درشت و ريز

\begin{tabular}{|c|c|c|c|c|c|c|c|}
\hline $\begin{array}{c}\text { Partial Eta } \\
\text { Squared }\end{array}$ & F & سارى م-r-r & سارى ا-r معنى - سطح & سطح معنى - & كفى بافت دار & درشى بافت دار & بدون كفى [1]" " \\
\hline
\end{tabular}

زمان رسيدن به اوج نيروى

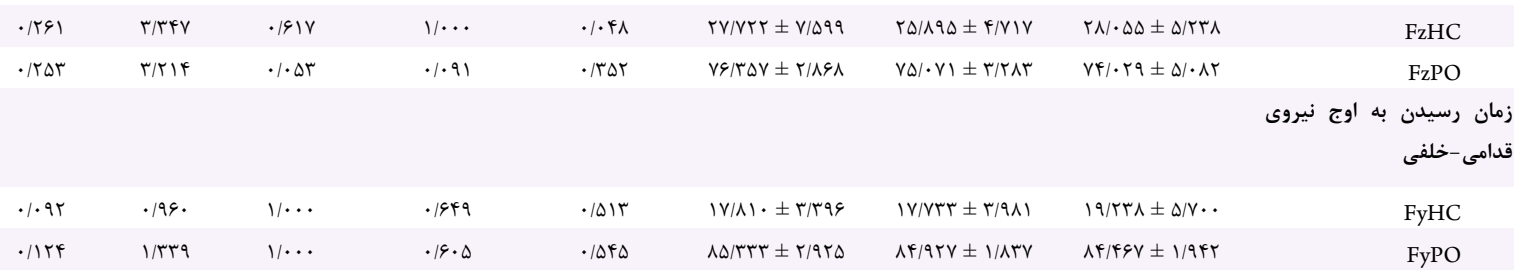

زمان رسيدن به اوج نيروى داخلى-خارجى

\begin{tabular}{|c|c|c|c|c|c|c|c|c|}
\hline a & $F / 901$ & $\cdot|| r \mid$ & $\cdot|\cdot r|$ & $1 / \cdots$ & 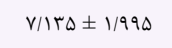 & $\Delta / V V r \pm r / r T r$ & $\Delta / F q r \pm r / q r \Lambda$ & FxHC \\
\hline $.1 .9 \mathrm{~V}$ & .1911 & $1 / \ldots$ & $\cdot|v V|$ & $1 / \cdots$ & $\Delta r / r I f \pm r \mid / F I r$ & $F q / .9 r \pm r r / . q 1$ & $F / / \wedge 9 \Delta \pm 19 / F \Delta \Lambda$ & FxPO \\
\hline
\end{tabular}


جدول F. مقادير كشتاور آزاد و نرخ بارَّارى (وزن بدن بر ثانيه) طى سه شرايط راه رفتن بدون كفى، با كفى بافت دار درشت و ريز

\begin{tabular}{|c|c|c|c|c|c|c|c|c|}
\hline $\begin{array}{l}\text { Partial Eta } \\
\text { Squared }\end{array}$ & $\mathbf{F}$ & سطح معنى - سارى & سارى ا-ب معى - س & سارى ا-1 معى- & كفى بافت دار ريز & كفى بافت دار & "بدون كفى [1]" & متغير \\
\hline & & & & & & & & كشتاور آزاد \\
\hline$\cdot 1 \cdot \Delta F$ & - IAVD & $\cdot / 9 \Delta$. & $1 / \cdots$ & $1 / \cdots$ & $-\cdot|r q T \pm \cdot / r T|$ & $-\cdot / T Y \Lambda \pm \cdot / T A V$ & $-\cdot / R 9 T \pm \cdot / 19 T$ & اوج منفى \\
\hline$\cdot / \cdot V$ & $\cdot / 1 / 91$ & $1 / \cdots$ & . & $1 / \cdots$ & $\cdot / 419 \pm \cdot / r \cdot r$ & $\cdot / 4 \cdot \Delta \pm \cdot / r \cdot \Delta$ & $\cdot / r V \Delta \pm \cdot / 1 V \Delta$ & اوج مثبت \\
\hline & & & & & & & & نرخ بارَذارى \\
\hline • / 998 & ए/१९९ & $1 / / \cdots$ & $1 / \cdots$ & .1 .91 & $r / 9 \Delta \cdot \pm 1 / 11 \mathrm{~V}$ & $r / l r I \pm 1 / \cdot r q$ & $r / \Lambda \cdot \uparrow \pm \cdot|\Lambda| \uparrow$ & عمودى \\
\hline
\end{tabular}

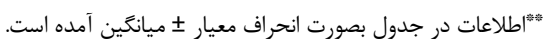

زمين در راستاى داخلى-خارجى در شرايط بدون كفى و استفاده از دو

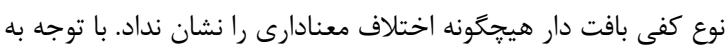

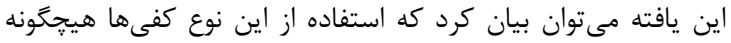
تاثيرى بر ميزان درد يا و زانو در سالمندان نداشته است. مطابق مطالعه

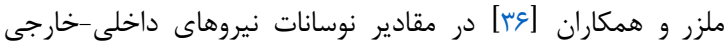

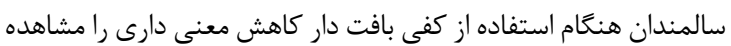

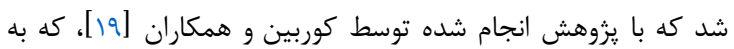

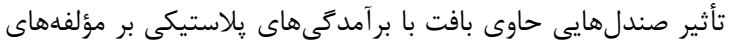

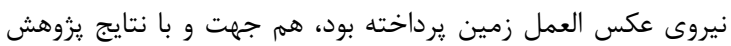

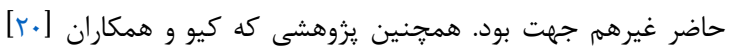

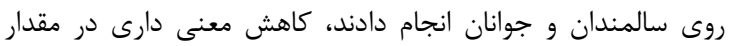

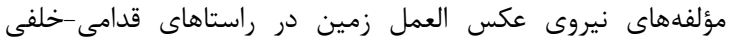
مشاهده كرد كه با نتايج حاضر همسو مىباشد. كاهش ميزان نيرو در

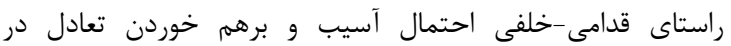

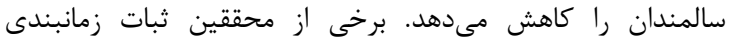

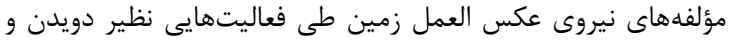

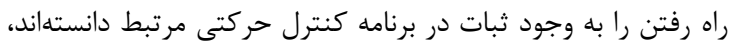

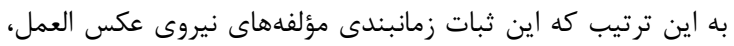

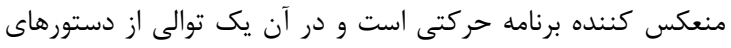

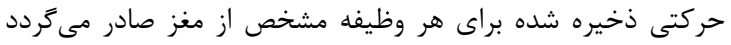

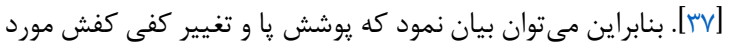

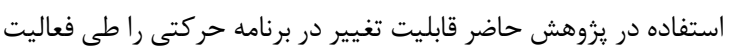

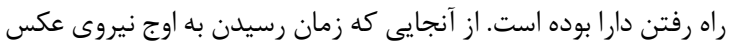

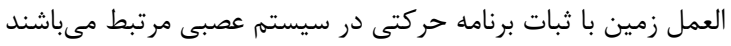

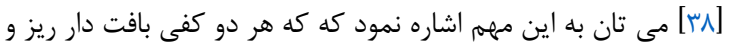

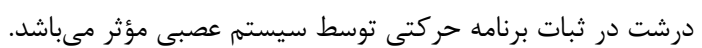

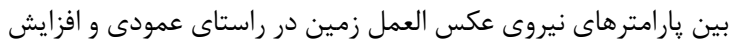

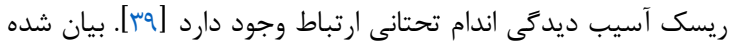

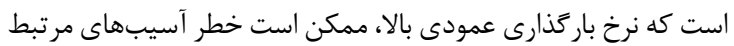

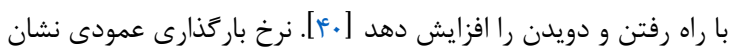

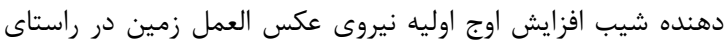

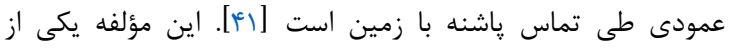

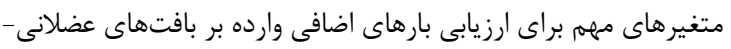

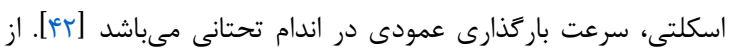

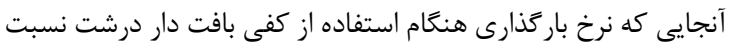

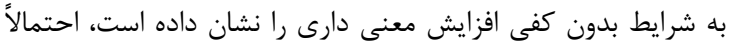

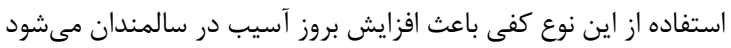

هدف يروهش حاضر بررسى اثر كفى بافت دار بر مؤلفهاى نيروى

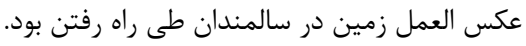

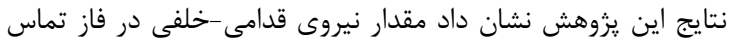

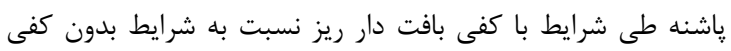

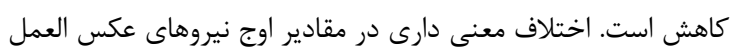

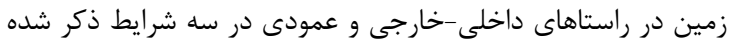

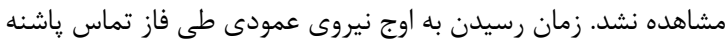

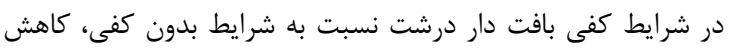

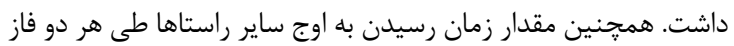

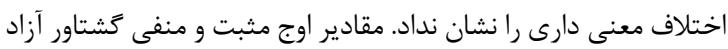
در سه شرايط معنى دار نبود. مطابق مطالعات كذشته ارتباط بهينه و مؤثر بين مارد مكانيزم هاى

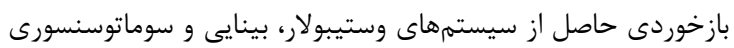

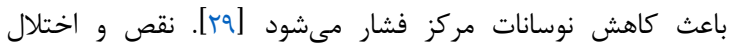

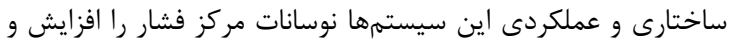

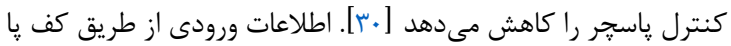

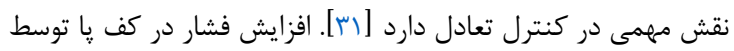

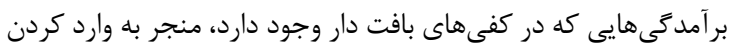

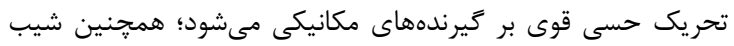

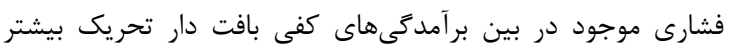

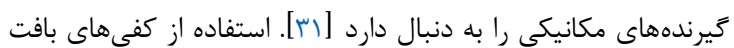

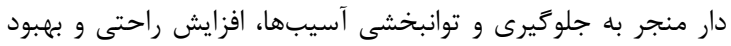

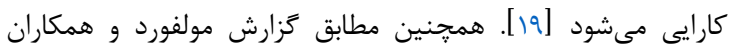

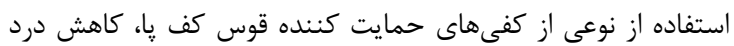

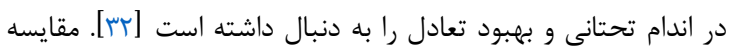

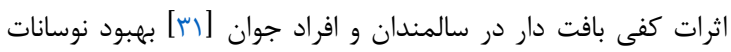

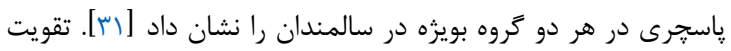

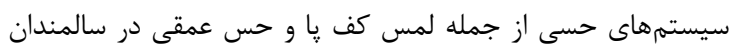

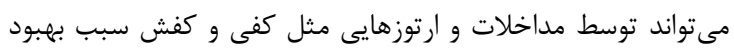

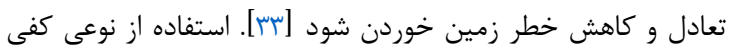

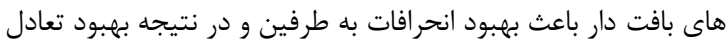

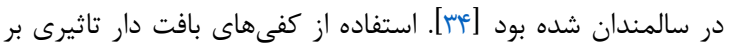
مقدار نوسانات و تعادل در سالمندان نداشت. نيروى عكس العمل زمين در جهت داخلى-خارجى احتمال بروز

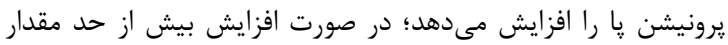

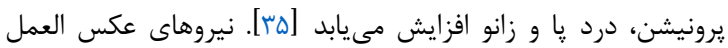


ملاحظات اخلاقى

اين يزوهش توسط كميته اخلاق دانشعاه علوم يزشكى اردبيل با شماره

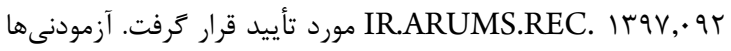

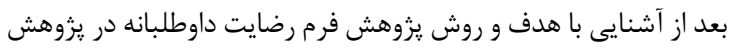

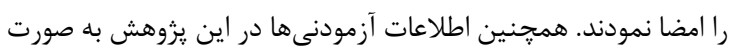
مخفى حفظ شد. حمايت مالى هيجگَونه حمايت مالى در يزوهش حاضر وجود نداشت. سهم نويسندكًان آقاى آيدين ولى زاده اورنج: ايده يزوهش از ايشان بوده و ورزن اوليه

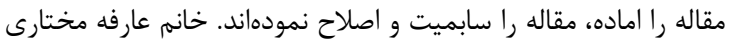

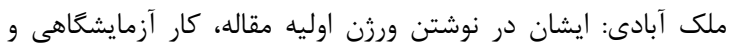

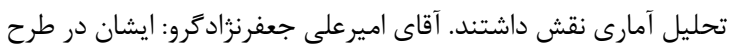

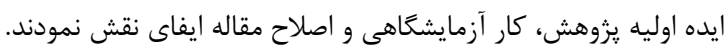

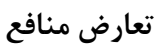
نويسنَان مقاله هيجگگُنه تعارض منافعى نداشتند. كاربرد عملى مقاله اين مطالعه در توانبخشى افراد سالمند مفيد است و مىتواند در كاهش نيروهاى عكس العمل زمين هنكام استفاده از كفى بافت دار طى دئ راه رفتن توصيه شود. سياسگذارى

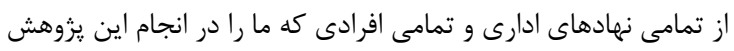

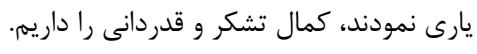

\section{References}

1. Robertson GE, Caldwell GE, Hamill J, Kamen G, Whittlesey S. Research methods in biomechanics: Human kinetics; 2018.

2. Cote KP, Brunet ME, II BMG, Shultz SJ. Effects of pronated and supinated foot postures on static and dynamic postural stability. 2005;40(1):41.

3. Kimura T, Kobayashi H, Nakayama E, Hanaoka M. Effects of aging on gait patterns in the healthy elderly. Anthropological Science. 2007;115(1):67-72. doi: 10.1537/ase. 060309 pmid: 29413451

4. Hortobágyi T, Finch A, Solnik S, Rider P, DeVita P. Association between muscle activation and metabolic cost of walking in young and old adults. J Gerontol Series Biomed Sci Med Sci. 2011;66(5):541-7. doi: 10.1093/gerona/glr008 pmid: 21345892

5. Farsi A, Ashayeri H, Mohamadzadeh S. Effect of six-week balance training on kinematic charactrestics during walking in female older adults. Salmand J. 2015;9(4):27887.

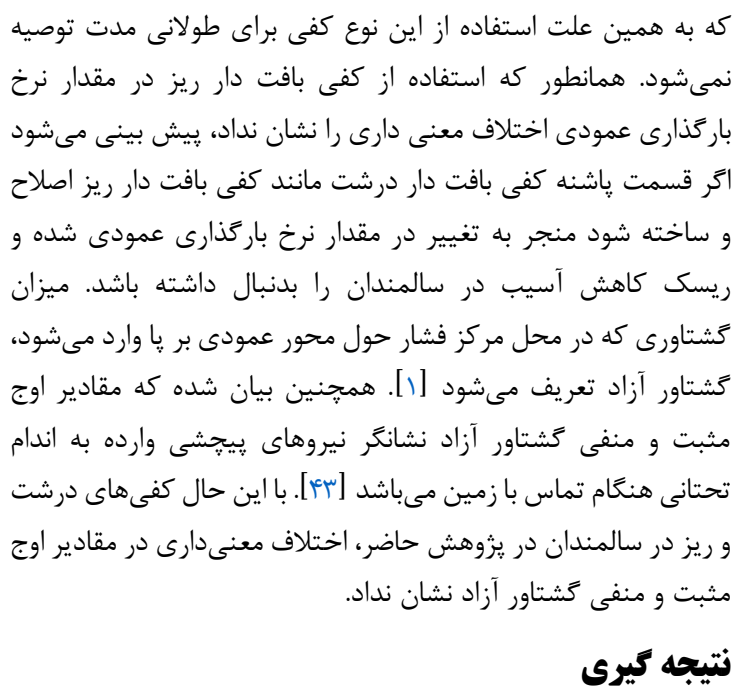

يافتههاى يزوهش حاضر نشان داد كه استفاده از كفى بافت دار ريز در

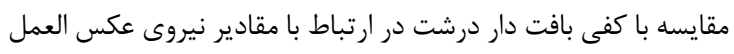

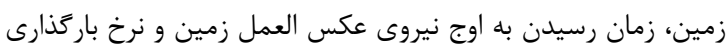

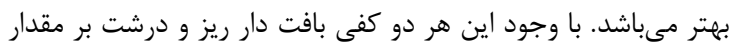

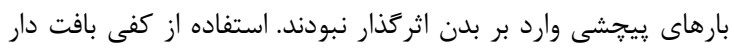

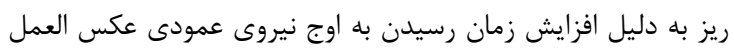

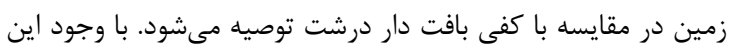

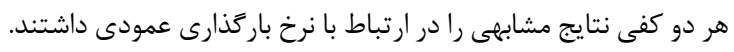

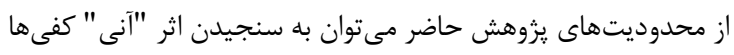

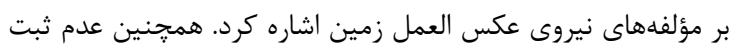

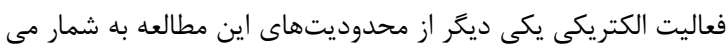

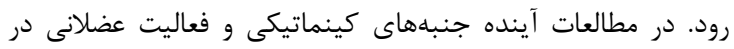

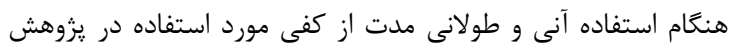
حاضر مورد بررسى قرار خيرد.

6. Farahpour N, Jafarnezhadgero AA, Allard P, Majlesi M. Muscle activity and kinetics of lower limbs during walking in pronated feet individuals with and without low back pain. J Electromyograph Kinesiol. 2018;39:35-41. doi: 10.1016/j.jelekin.2018.01.006 pmid: 29413451

7. Hatton AL, Dixon J, Rome K, Martin D. Standing on textured surfaces: effects on standing balance in healthy older adults. Age Ageing. 2011;40(3):363-8. doi: 10.1093/ageing/afr026 pmid: 21450692

8. Melzer I, Benjuya N, Kaplanski J. Postural stability in the elderly: a comparison between fallers and non-fallers. Age Ageing. 2004;33(6):602-7. doi: 10.1093/ageing/afh218 pmid: 15501837

9. Kerzman H, Chetrit A, Brin L, Toren O. Characteristics of falls in hospitalized patients. J Advance Nurs. 2004;47(2):223-9. doi: 10.1111/j.13652648.2004.03080.x

10. Berry SD, Miller RR. Falls: epidemiology, pathophysiology, and relationship to fracture. Curr 
Osteoporosis Report. 2008;6(4):149-54. doi: 10.1007/s11914-008-0026-4 pmid: 19032925

11. Brooshak N, Asadi M, Hosseini SH. Comparison of the Percentage Stance and Swing Phases and Ground Reaction Force between Young and Older Adults during Walking at different speeds. J Sport Biomechanic. 2017;3(2):5-14.

12. Qiu F, Cole MH, Davids KW, Hennig EM, Silburn PA, Netscher $\mathrm{H}$, et al. Effects of textured insoles on balance in people with Parkinson's disease. J PloS one. 2013;8(12):e83309. doi: 10.1371/journal.pone.0083309 pmid: 24349486

13. Kerrigan DC, Lelas JL, Goggins J, Merriman GJ, Kaplan RJ, Felson DT. Effectiveness of a lateral-wedge insole on knee varus torque in patients with knee osteoarthritis. Archive Physic Med Rehabilit. 2002;83(7):889-93. doi: 10.1053/apmr.2002.33225 pmid: 12098144

14. McGrath D, Judkins TN, Pipinos II, Johanning JM, Myers SA. Peripheral arterial disease affects the frequency response of ground reaction forces during walking. J Clinic Biomechanic. 2012;27(10):1058-63. doi: 10.1016/j.clinbiomech.2012.08.004 pmid: 22967739

15. Hatton AL, Dixon J, Rome K, Martin D. Standing on textured surfaces: effects on standing balance in healthy older adults. J Age Ageing. 2011;40(3):363-8. doi: 10.1093/ageing/afr026 pmid: 21450692

16. Salari-Moghaddam F, Sadeghi-Demneh E, Ja'farian FS. The Effects of Textured Insole on Ankle Proprioception and Balance in Subjects with the Risk of Falling. J Arch Rehabilit. 2015;16(1):58-65.

17. Palluel E, Olivier I, Nougier V. The lasting effects of spike insoles on postural control in the elderly. J Behav Neurosci. 2009;123(5):1141. doi: 10.1037/a0017115 pmid: 19824780

18. Wilson ML, Rome K, Hodgson D, Ball P. Effect of textured foot orthotics on static and dynamic postural stability in middle-aged females. J Gait Posture. 2008;27(1):36-42. doi: 10.1016/j.gaitpost.2006.12.006 pmid: 17267222

19. Corbin DM, Hart JM, McKeon PO, Ingersoll CD, Hertel $\mathrm{J}$. The effect of textured insoles on postural control in double and single limb stance. J Sport Rehabilit. 2007;16(4):363-72. doi: 10.1123/jsr.16.4.363 pmid: 18246902

20. Qiu F, Cole MH, Davids KW, Hennig EM, Silburn PA, Netscher $\mathrm{H}$, et al. Enhanced somatosensory information decreases postural sway in older people. J Gait Posture. 2012;35(4):630-5. doi: 10.1016/j.gaitpost.2011.12.013 pmid: 22245163

21. Purpirali M, Kalantari M, Taghizadeh G, Mehdizadeh H. Effect of Textured insole on the complexity of center of pressure sway in faller older adults. J Rehabilitat Med. 2015;4(2):29-36.

22. McKeon PO, Stein AJ, Ingersoll CD, Hertel J. Altered plantar-receptor stimulation impairs postural control in those with chronic ankle instability. J Sport Rehabilit. 2012;21(1):1-6. doi: 10.1123/jsr.21.1.1 pmid: 22318247
23. Milner CE, Davis IS, Hamill J. Free moment as a predictor of tibial stress fracture in distance runners.J Biomechanic. 2006;39(15):2819-25. doi: 10.1016/j.jbiomech.2005.09.022 pmid: 16289078

24. Hoseini Y, Farahpour NJ. The Effects of Arch Support Insole on Ground Reaction Force, Impulse and Loading Rate during Double-Leg Landing. J Paramed Sci Rehabilitat. 2018;7(3):46-53.

25. World Medical Association. "Ethical principles for medical research involving human subjects," Declaration of Helsinki 2004. Available from: www.wma. net/e/policy/b3. htm.

26. Begg RK, Sparrow WA, Lythgo ND. Time-domain analysis of foot-ground reaction forces in negotiating obstacles. J Gait Posture. 1998;7(2):99-109. doi: 10.1016/S0966-6362(97)00039-8

27. Shapiro DC, Zernicke RF, Gregor RJ, Diestel JD. Evidence for generalized motor programs using gait pattern analysis. J Motor Behav. 1981;13(1):33-47. doi: 10.1080/00222895.1981.10735235 pmid: 15201130

28. Cohen J. Statistical power analysis for the behavioral sciences: Routledge; 2013.

29. Costa M, Priplata AA, Lipsitz LA, Wu Z, Huang NE, Goldberger $\mathrm{AL}$, et al. Noise and poise: enhancement of postural complexity in the elderly with a stochasticresonance-based therapy. EPL (Europhysics Letters). 2007;77(6):68008. doi: 10.1209/0295-5075/77/68008 pmid: 17710211

30. Duarte M, Sternad D. Complexity of human postural control in young and older adults during prolonged standing. Experiment Brain Res. 2008;191(3):265-76. doi: $10.1007 /$ s00221-008-1521-7 pmid: 18696056

31. Qiu F, Cole MH, Davids KW, Hennig EM, Silburn PA, Netscher $\mathrm{H}$, et al. Enhanced somatosensory information decreases postural sway in older people. Gait Posture. 2012;35(4):630-5. doi: 10.1016/j.gaitpost.2011.12.013 pmid: 22245163

32. Mulford D, Taggart HM, Nivens A, Payrie C. Arch support use for improving balance and reducing pain in older adults. J Appl Nurs Res. 2008;21(3):153-8. doi: 10.1016/j.apnr.2006.08.006 pmid: 18684409

33. Hijmans JM, Geertzen JHB, Dijkstra PU, Postema K. A systematic review of the effects of shoes and other ankle or foot appliances on balance in older people and people with peripheral nervous system disorders. Gait Posture. 2007;25(2):316-23. doi: 10.1016/j.gaitpost.2006.03.010 pmid: 16687248

34. Stacoff A, Kramers-de Quervain I, Dettwyler M, Wolf P, List R, Ukelo T, et al. Biomechanical effects of foot orthoses during walking. Foot. 2007;17(3):143-53. doi: 10.1016/j.foot.2007.02.004

35. Rodrigues $\mathrm{P}, \mathrm{TenBroek} \mathrm{T}, \mathrm{Hamill} \mathrm{J}$. Runners with anterior knee pain use a greater percentage of their available pronation range of motion. J Appl Biomechanic. 2013;29(2):141-6. doi: 10.1123/jab.29.2.141 pmid: 23645486

36. Melzer I, Benjuya N, Kaplanski J. Postural stability in the elderly: a comparison between fallers and non-fallers. J 
Age Ageing. 2004;33(6):602-7. doi: 10.1093/ageing/afh218 pmid: 15501837

37. Begg RK, Sparrow WA, Lythgo ND. Time-domain analysis of foot-ground reaction forces in negotiating obstacles. Gait Posture. 1998;7(2):99-109. doi: 10.1016/S0966-6362(97)00039-8

38. Jafarnezhadgero AA, Majlesi M, Azadian E. Gait ground reaction force characteristics in deaf and hearing children. Gait Posture. 2017;53:236-40. doi: 10.1016/j.gaitpost.2017.02.006 pmid: 28219845

39. Messier SP, Martin DF, Mihalko SL, Ip EH, DeVita P, Cannon DW, et al. A 2-year Prospective Observational Study To Determine The Etiologic Factors Associated With Overuse Running Injuries: 652 June 1, 2. Med Sci Sport Exercise. 2016;48(5S):170-1. doi: 10.1249/01.mss.0000485515.20061.df

40. Zadpoor AA, Nikooyan A. The relationship between lower-extremity stress fractures and the ground reaction force: a systematic review. Clinic Biomechanic. 2011;26(1):23-8. doi: 10.1016/j.clinbiomech.2010.08.005 pmid: 20846765

41. Stacoff A, Diezi C, Luder G, Stüssi E, Kramers-de Quervain IA. Ground reaction forces on stairs: effects of stair inclination and age. Gait Posture. 2005;21(1):24-38. doi: $10.1016 /$ j.gaitpost.2003.11.003 pmid: 15536031

42. de Oliveira Silva D, Briani RV, Pazzinatto MF, Ferrari D, Aragão FA, de Azevedo FM. Reduced knee flexion is a possible cause of increased loading rates in individuals with patellofemoral pain. Clinic Biomechanic. 2015;30(9):971-5.

doi:

10.1016/j.clinbiomech.2015.06.021 pmid: 26169602

43. Yang PF, Sanno M, Ganse B, Koy T, Brüggemann GP, Müller LP, et al. Torsion and antero-posterior bending in the in vivo human tibia loading regimes during walking and running. PLoS One. 2014;9(4):e94525. doi: 10.1371/journal.pone.0094525 pmid: 24732724 